PROCEEDINGS OF THE

AMERICAN MATHEMATICAL SOCIETY

Volume 135, Number 2, February 2007, Pages 393-395

S 0002-9939(06)08472-3

Article electronically published on August 21, 2006

\title{
COMPLEMENTED INVARIANT SUBSPACES AND INTERPOLATION SEQUENCES
}

\author{
EVGUENI DOUBTSOV
}

(Communicated by Joseph A. Ball)

\begin{abstract}
It is shown that the invariant subspace of the Bergman space $A^{p}$ of the unit disc, generated by a finite union of Hardy interpolation sequences, is complemented in $A^{p}$.
\end{abstract}

Let $\mathbb{D}$ be the open unit disc of the complex plane $\mathbb{C}$, and let $H^{p}=H^{p}(\mathbb{D})$ denote the usual Hardy space. For $0<p<\infty$ the Bergman space $A^{p}$ consists of analytic functions $f$ on $\mathbb{D}$ such that

$$
\|f\|_{p}^{p}=\int_{\mathbb{D}}|f(z)|^{p} d A(z)<\infty,
$$

where $d A$ is area measure on $\mathbb{C}$ normalized so that $A(\mathbb{D})=1$.

A closed subspace $I$ of $A^{p}$ is said to be invariant if $z f(z) \in I$ for all $f \in I$.

A sequence $Z=\left\{a_{j}\right\}$ of points in $\mathbb{D}$ is called an $A^{p}$ zero set if there exists a non-zero function $f \in A^{p}$ such that $f$ vanishes on $Z$, counting multiplicities. Every $A^{p}$ zero set generates an invariant subspace $I_{Z}^{p}$ consisting of all functions in $A^{p}$ that vanish on $Z$, counting multiplicities.

Recently, Korenblum and Zhu [4] exhibited several classes of complemented invariant subspaces $I$ in $A^{p}$. By definition, $I$ is complemented in $A^{p}$ if there exists a bounded projection $Q$ from $A^{p}$ onto $I$.

In particular, Korenblum and Zhu considered the subspaces $I_{Z}^{p}$ for a special class of zero sequences $Z$. Recall that a sequence $\left\{a_{j}\right\}$ of distinct points in the disc is an $A^{p}\left(H^{p}\right)$ interpolation sequence if for every sequence $\left\{w_{j}\right\} \subset \mathbb{C}$ with

$$
\sum\left(1-\left|a_{j}\right|^{2}\right)^{2}\left|w_{j}\right|^{p}<\infty \quad\left(\sum\left(1-\left|a_{j}\right|^{2}\right)\left|w_{j}\right|^{p}<\infty\right)
$$

there exists a function $f \in A^{p}\left(f \in H^{p}\right)$ such that $f\left(a_{j}\right)=w_{j}$ for all $j$. Recall that every $H^{p}$ interpolation sequence is an $A^{p}$ interpolation set (see, e.g., [5]); the converse implication does not hold. Further results about the interpolation sequences can be found in the monographs [3] and [2].

Theorem 1 (Korenblum and Zhu [4]). Suppose that $0<p<\infty$ and $Z$ is an $A^{p}$ interpolation sequence. Then the invariant subspace $I_{Z}^{p}$ is complemented in $A^{p}$.

Received by the editors August 10, 2005 and, in revised form, August 22, 2005.

2000 Mathematics Subject Classification. Primary 47A15; Secondary 30D55, 30H05.

Key words and phrases. Bergman space, Hardy space, invariant subspace.

This paper was written while the author enjoyed the hospitality of the Chalmers University of Technology.

(C)2006 American Mathematical Society Reverts to public domain 28 years from publication 
In the present paper we consider a different class of zero sets $Z$ (with possible multiplicities).

Theorem 2. Suppose that $0<p<\infty$ and $Z$ is a finite union of $H^{p}$ interpolation sequences. Then the invariant subspace $I_{Z}^{p}$ is complemented in $A^{p}$.

Proof. Let $Z=\bigcup_{k=1}^{n} Z_{k}$, where $Z_{k}$ is an $H^{p}$ interpolation sequence, $k=1,2, \ldots, n$. Observe that $Z_{k}$ is an $A^{p}$ interpolation sequence. Therefore, by Theorem 1, there exists a bounded linear operator (a projection) $Q_{k}$ from $A^{p}$ onto $I_{Z_{k}}^{p}$ such that $Q_{k}^{2}=Q_{k}$.

Consider the Blaschke products

$$
B_{m}(z)=\prod_{a_{j} \in Z(m)} \frac{\left|a_{j}\right|}{a_{j}} \frac{a_{j}-z}{1-z \bar{a}_{j}}, \quad m=1,2, \ldots, n,
$$

where $Z(m)=\bigcup_{k=1}^{m} Z_{k}$ (as usual, if $a_{j}=0$, then the quotient $\left|a_{j}\right| / a_{j}$ is replaced by -1$)$. Let $B_{m} A^{p}$ denote the set of all multiples of $B_{m}$ by functions in $A^{p}$. Since $Z(m)$ is a finite union of $H^{p}$ interpolation sequences, we have

$$
\begin{aligned}
B_{m} A^{p} & =I_{Z(m)}^{p}, \\
\left\|f / B_{m}\right\|_{p} & \leq \operatorname{const}(m)\|f\|_{p} \text { for every function } f \in I_{Z(m)}^{p}
\end{aligned}
$$

(see, e.g., 2], Sections 4.5 and 4.6).

Given $f \in I_{Z(m-1)}^{p}, m=2,3, \ldots, n$, put

$$
T_{m} f=B_{m-1} Q_{m}\left(f / B_{m-1}\right)
$$

(cf. [1, where such operators are used in the $H^{p}$ setting). Finally, define

$$
Q=T_{n} T_{n-1} \ldots T_{2} Q_{1}
$$

By (11) and (2), $Q$ is a bounded linear operator from $A^{p}$ onto $I_{Z(n)}^{p}=I_{Z}^{p}$ such that $Q^{2}=Q$. In other words, $I_{Z}^{p}$ is complemented in $A^{p}$.

\section{ADDED IN PROOF}

A different proof of Theorem 2 can be obtained with the help of interpolation techniques developed in the following paper: Hartmann A., Traces of certain classes of holomorphic functions on finite unions of Carleson sequences, Glasg. Math. J. 41 (1999), no. 1, 103-114. The author is grateful to Andreas Hartmann for this reference.

\section{REFERENCES}

[1] Cohn W.S., Complemented invariant subspaces of $H^{p}, 0<p<1$, and the Hahn-Banach extension property, Proc. Amer. Math. Soc. 102 (1988), no. 1, 121-124. MR0915728 (88k:46063)

[2] Duren P., Schuster A., Bergman spaces, Mathematical Surveys and Monographs, vol. 100, Amer. Math. Soc., Providence, RI, 2004. MR2033762 (2005c:30053)

[3] Hedenmalm H., Korenblum B., Zhu K., Theory of Bergman spaces, Graduate Texts in Mathematics, vol. 199, Springer-Verlag, New York, 2000. MR.1758653 (2001c:46043) 
[4] Korenblum B., Zhu K., Complemented invariant subspaces in Bergman spaces, J. London Math. Soc. (2) 71 (2005), no. 2, 467-480. MR2122439 (2006a:46031)

[5] Zhu K.H., Interpolating sequences for the Bergman space, Michigan Math. J. 41 (1994), no. 1, 73-86. MR:1260609 (94m:46045)

St. Petersburg Department, V.A. Steklov Institute of Mathematics, Fontanka 27, 191023 St. Petersburg, Russia

E-mail address: dubtsov@pdmi.ras.ru 